\title{
Increased incidence of respiratory symptoms among female woodworkers exposed to dry wood
}

\author{
G. Jacobsen*, V. Schlünssen*, I. Schaumburg* and T. Sigsgaard*
}

ABSTRACT: The aim of the present study was to investigate the occurrence of new respiratory symptoms in relation to wood dust exposure in a 6-yr follow-up study.

A total of 1,377 woodworkers (1,137 males; 240 females) and 297 reference workers (137 males; 160 females) participated. Data on respiratory symptoms, employment and smoking habits were collected. Wood dust exposure was assessed from baseline dust measurements, and cumulative wood dust exposure was assessed by study-specific job exposure matrices and exposure time.

The geometric mean (geometric SD) dust level decreased during the study period from 0.94 (2.1) to $0.60(1.6) \mathrm{mg} \cdot \mathrm{m}^{-3}$. Adjusted analysis revealed positive associations for cumulative incidence proportion of chronic bronchitis and daily coughing for female woodworkers versus female reference workers. The cumulative incidence proportion of daily coughing and chronic bronchitis were found to be associated with baseline wood dust exposure in a dose-dependent manner. The odds ratio (95\% confidence interval) for daily coughing (with reference to the lowest exposure quartile) was $1.6(0.6-4.3), 3.2(0.9-6.8)$ and $3.8(1.5-9.7)$, respectively, in the second and third lowest and the highest quartile. The figures for chronic bronchitis were, accordingly, 2.3 (0.414.5), 3.0 (0.5-18.7) and $6.0(1.2-28.8)$.

In conclusion, female woodworkers in this low exposure cohort showed an increased incidence of coughing and bronchitis, whereas no relations to wood dust exposure were seen for male woodworkers.

KEYWORDS: Asthma, incidence, occupation, respiratory symptoms, sex, wood dust

W ood dust has long been associated with a variety of respiratory diseases, and is an established cause of sinonasal cancer

[1]. Regarding nonmalignant respiratory diseases, the majority of knowledge is based on cross-sectional studies, in which increased prevalences of asthma, chronic bronchitis, nasal symptoms and eye symptoms have been reported [2, 3]. In addition, studies have shown an increased cross-shift decline in lung function. In some studies, positive dose-response relations between wood dust exposure and respiratory impairment have been revealed [4,5].

Western red cedar is the only type of wood to have been thoroughly studied, and the principal agent causing occupational asthma, plicatic acid, has been revealed [6].

In Canada, the incidence of western red cedar asthma was estimated from a cross-sectional study [7], whereas, for woodworkers exposed to other wood types, the incidence of respiratory symptoms has mainly been estimated from national registers on employment and use of asthma medication $[8,9]$. To date, no follow-up studies on respiratory diseases have been performed in the dry wood industry.

Exposure assessments in epidemiological studies in the wood industry are often based on dust measurements sampled for other purposes, or performed with qualitative data supplemented by a limited number of measurements. In addition, most previous studies have been carried out on smaller study populations, in which confounders, such as smoking, height and age, could not be dealt with sufficiently.

In the European Union (EU) $\sim 3.6$ million $(2.0 \%$ of the working population) workers have been estimated to be occupationally exposed to wood dust. Of these, 700,000 are exposed in the furniture industry. In Denmark, 70,000 (3.3\% of the working population) workers are exposed to wood dust, with 15,000 workers employed in the furniture industry $[10,11]$. In the Danish furniture industry, the wood types processed are

\section{AFFILIATIONS}

*Dept of Environmental and Occupational Medicine, School of Public Health, Aarhus University, and \#Neuro Centre, Aarhus Sygehus, Aarhus University Hospital, Aarhus, Denmark.

\section{CORRESPONDENCE}

G. Jacobsen

Dept of Environmental and

Occupational Medicine

School of Public Health

Aarhus University

Bartholins allé 2

8000 Aarhus C

Denmark

Fax: 4589426199

E-mail: gitte.jacobsen@dadlnet.dk

Received:

March 272008

Accepted after revision:

February 022009

SUPPORT STATEMENT

This study was supported by Viborg

County (Viborg, Denmark) and the

Danish Work Environment

Foundation, the Danish Medical

Research Council, the Health

Insurance Fund and the Danish Lung

Association (all Copenhagen,

Denmark).

STATEMENT OF INTEREST

None declared. 
mainly softwoods, predominantly pine and wood composites, i.e. particle board (or chipboard) and medium-density fibreboard $[11,12]$.

The aim of the present follow-up study was to reveal whether an increased incidence of asthma and respiratory symptoms were present on exposure to the wood types and exposure levels encountered in the modern Danish furniture industry.

\section{MATERIALS AND METHODS}

\section{Study population}

The baseline study population was identified in a crosssectional study performed during 1997-1998, which has been described elsewhere [3]. In brief, 86 factories with more than four employees situated in Viborg County were identified. All factories with $>20$ employees were asked to participate (45 out of 48 accepted), and, additionally, a random sample was drawn of factories with 5-20 employees (nine out of 38 accepted, all nine accepted). A total of 54 factories participated in the study. The study population was workers employed in the woodworking, assembly and stock departments of these factories. Additionally, three factories (two producing refrigerators and one producing hearing aids) in the same area were selected as reference.

A questionnaire was returned by 2,032 woodworkers and 474 control subjects, who were defined as the population for follow-up. Follow-up took place during the period April 2003 to April 2005. Visits to 52 furniture factories (of which 38 also participated at baseline) initially resulted in contact with 937 individuals from the exposed group and 215 reference workers. The remaining subjects were contacted by letter and

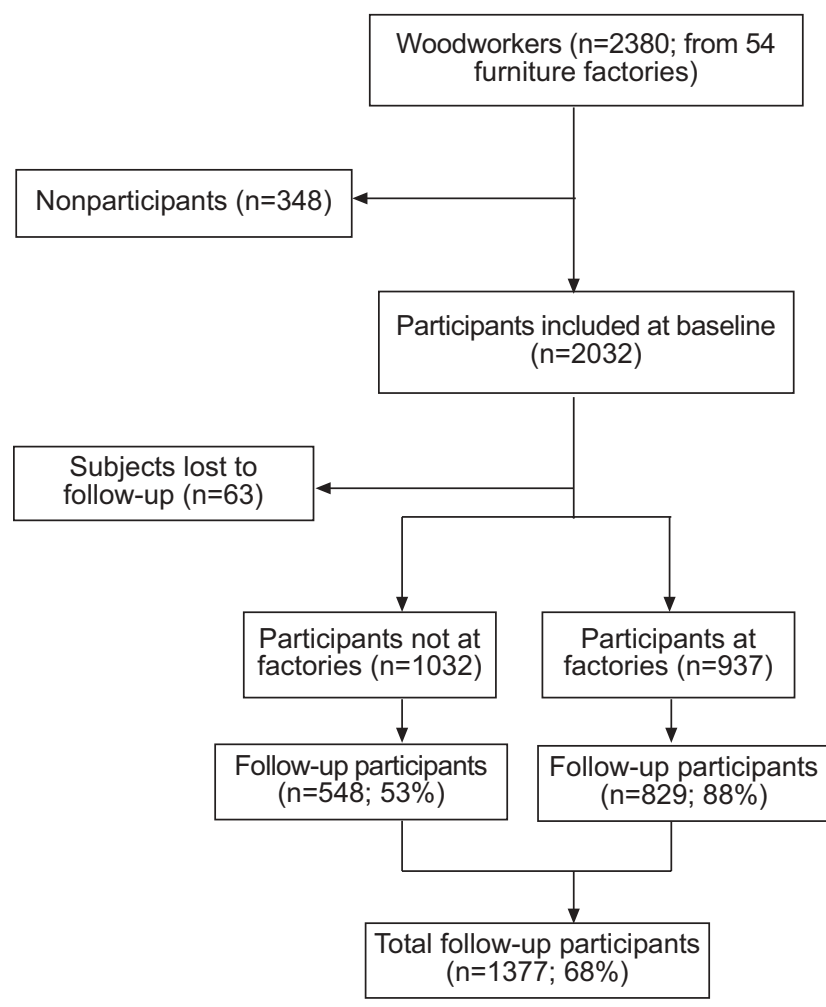

invited to participate. In total 1,377 (68\%) woodworkers and 297 (63\%) reference workers participated at follow-up. A flow chart of the study is shown in figure 1.

All participants gave informed consent, and the protocol was approved by the Ethics Committee for Viborg County (Aalborg, Denmark)

\section{Health outcomes}

A modified UK Medical Research Council questionnaire [13], including key European Community Respiratory Health Survey questions on asthma [14] and additional questions on allergy, coughing, asthma, rhinitis, smoking and occupational history, was distributed at baseline and at follow-up [3].

Ever-asthma was defined as current or ever self-reported asthma. Asthma symptoms was defined if the subject answered yes to at least one group A question and two or more group B questions or at least two group A questions (table 1) [3, 15]. Chronic bronchitis was defined as expectoration on most days during $\geqslant 3$ months consecutively for $>2$ yrs consecutively according to UK Medical Research Council guidelines [13]. Incident cases were defined among individuals without the symptom at baseline.

Asthma symptoms at baseline was validated, where a sensitivity of $75 \%$ and a specificity of $61 \%$ were found against objective indications of asthma (bronchial hyperresponsiveness, positive reversibility test or increased peak expiratory flow variability) [16].

Lung function testing was performed at baseline and followup, and has been described previously [3, 12]. In brief, forced

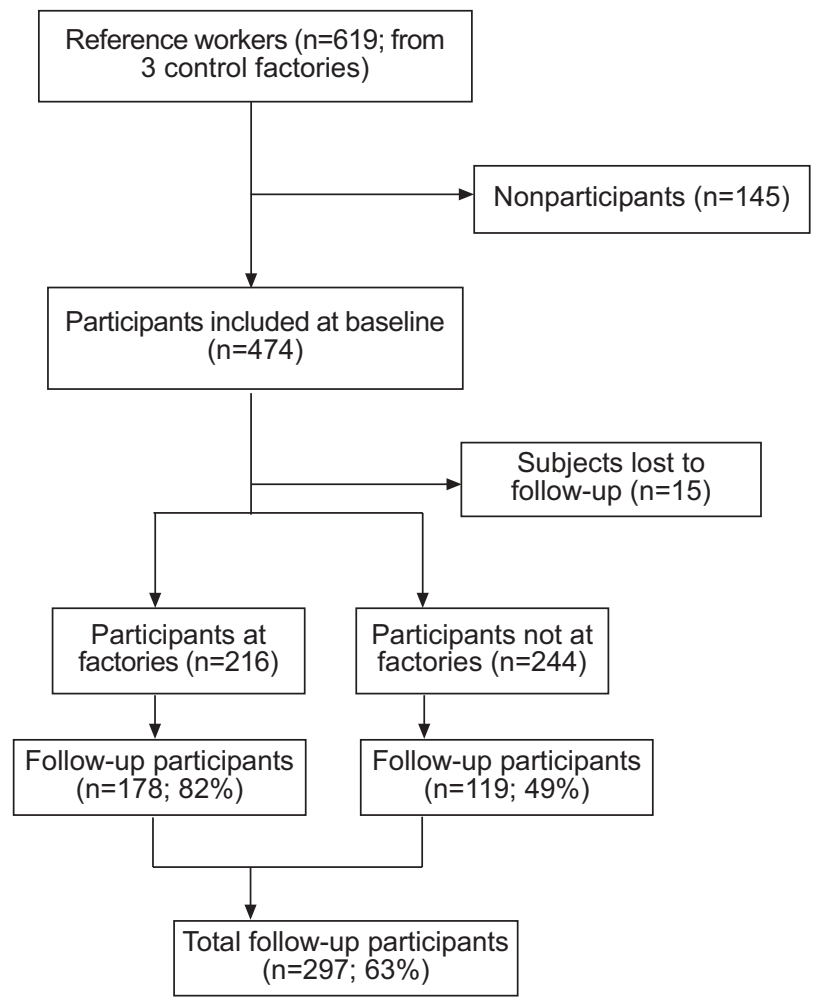

FIGURE 1. Flow chart of the study. 


\begin{tabular}{|c|c|}
\hline Group A & Group B \\
\hline Do you have asthma? & Do you ever wake in the night with chest tightness? \\
\hline Have you ever had asthma? & Do you wake in the night wheezing? \\
\hline \multirow[t]{6}{*}{ Do you ever wheeze? } & Do you cough when you wake up in the morning? \\
\hline & Do you wheeze because of exposure to cold air? \\
\hline & Do you wheeze when you exercise? \\
\hline & Do you wheeze because of exposure to pollen? \\
\hline & Do you wheeze because of exposure to animals? \\
\hline & Do you use asthma drugs? \\
\hline
\end{tabular}

\#: asthma symptoms were regarded as present when subjects answered yes to at least one group A question and two or more group B questions

expiratory volume in $1 \mathrm{~s}$ (FEV1), forced vital capacity, height and weight were measured and used to calculate the percentage of the predicted values at baseline and follow-up and the annual change in lung function.

Blood sampling was performed in a subpopulation at followup. Atopic status was available for 935 woodworkers and 195 controls, and was defined as a positive (specific immunoglobulin E to 12 common respiratory allergens) Phadiatop ${ }^{\circledR}$ (Phadia, Uppsala, Sweden; analyses performed at Phadia, Alleroed, Denmark) result [17].

\section{Exposure assessment}

Personal dust samplings were carried out at baseline and follow-up using the passive dust monitors described previously [18]. In brief, the method is based on measuring light extinction before and after sampling on transparent sticky foils, reported as dust-covered foil area and conver-ted to equivalent inhalable dust by linear regression models based on earlier and present calibration measurements $[11,19]$. In order to calculate cumulative wood dust exposure during the follow-up period, two job exposure matrices based on factory size and task were constructed at baseline (12 groups, 2,217 measurements and 1,581 individuals) and follow-up (seven groups, 1,355 measurements and 1,044 individuals). Individual cumulative exposure was estimated from the exposure level at baseline for half the period (until 2001), the exposure level at follow-up for the remaining period and the duration of exposure in the follow-up period [12].

Terpene ( $\alpha$-pinene, $\beta$-pinene and $\Delta$-3-carene) measurements on a random $25 \%$ sample of workers employed in 17 factories primarily using pine was performed at follow-up $(n=161)$. A personal passive monitor (SKC 575-003 with Anasorb 727; SKC, Inc., Eighty Four, PA, USA) [20] was used.

A personal passive SKC UMEx 100 diffusion sampler (SKC, Inc.) [21] was used to collect 24 formaldehyde measurements in 10 factories, using a worst case strategy.

Smokers were defined as present smokers or ex-smokers for $>2$ yrs prior to the baseline study.

\section{Analysis}

When data were normally distributed, mean \pm SD is reported. When data were log-normally distributed, geometric mean (geometric SD) is stated. When data were neither normally nor log-normally distributed, median (range) is reported.

Univariate analyses of baseline characteristics of the cohort were undertaken for categorical variables using Chi-squared tests. For continuous variables, independent-sample t-tests were used.

The cumulative incidence proportion of symptoms stratified by sex and smoking was analysed using Chi-squared tests. In further analyses, association between exposure and the cumulative incidence proportion of symptoms was analysed by multiple logistic regression. The analyses were stratified by sex and adjusted for potential confounders. In order to test for trends across exposure groups, regression analyses were performed treating exposure groups as a continuous variable.

Unless otherwise stated, a significance level of 5\% was used.

\section{RESULTS}

The participation rate during follow-up was $68 \%$ for woodworkers and $63 \%$ for reference workers. At baseline, participants in general were older than nonparticipants, and smoking was more prevalent among nonparticipants. A tendency was seen for nonparticipants to show more symptoms at baseline. Participating woodworkers had a longer history of employment in the wood industry. The mean tenure at baseline was $10 \mathrm{yrs}, 10.8 \pm 9.3 \mathrm{yrs}$ (range $0.1-45 \mathrm{yrs}$ ) for males and $4.9 \pm 4.8$ yrs (range $0.1-29$ yrs) for females [16]. No significant difference in baseline wood dust exposure was revealed between participants and nonparticipants (table 2).

Participating woodworkers were slightly older than participating reference workers. The mean \pm SD age was $38.3 \pm 11.2$ (range 17-68) yrs for males and 37.0 \pm 9.6 (range 17-64) yrs for females. No significant differences in symptom status or smoking at baseline between woodworkers and reference workers of either sex were seen (table 2).

A higher percentage of both woodworkers and reference workers encountered at the factories participated compared 
TABLE 2 Demographic characteristics of cohort at baseline

\begin{tabular}{|c|c|c|c|c|c|c|c|c|}
\hline & \multicolumn{2}{|c|}{ Woodworkers } & \multicolumn{2}{|c|}{ Reference workers } & \multicolumn{2}{|c|}{ Woodworkers } & \multicolumn{2}{|c|}{ Reference workers } \\
\hline Subjects ${ }^{\#} \mathrm{n}$ & 1137 & 528 & 137 & 124 & 240 & 127 & 160 & 53 \\
\hline Daily coughing at BL & $338(30.8)$ & $178(35.8)$ & $41(31.3)$ & $43(38.1)$ & $71(32.0)$ & $50(41.3)$ & $37(24.5)$ & $19(36.5)$ \\
\hline Chronic bronchitis at BL & $102(10.3)$ & $47(10.4)$ & $12(10.5)$ & $14(13.7)$ & $10(5.0)$ & $7(6.5)$ & $9(6.6)$ & $3(6.7)$ \\
\hline Ever-asthma at BL & $60(5.4)$ & $33(6.7)$ & $8(6.1)$ & $8(6.7)$ & $16(7.0)$ & $11(8.7)$ & $14(9.1)$ & $3(5.8)$ \\
\hline Asthma symptoms at $\mathrm{BL}^{+}$ & $154(13.6)$ & 79 (15.3) & $18(13.3)$ & $24(19.8)$ & $38(16.2)$ & $21(16.5)$ & $20(12.6)$ & $9(17.0)$ \\
\hline Hay fever at BL & $145(13.3)$ & $71(14.1)$ & $21(15.8)$ & $17(14.3)$ & $29(12.9)$ & $17(14.3)$ & $22(14.5)$ & $7(14.3)$ \\
\hline Time in woodwork at BL yrs & $11.9 \pm 9.6^{*}$ & $8.3 \pm 8.1$ & & & $5.7 \pm 5.0^{\star}$ & $3.3 \pm 4.1$ & & \\
\hline
\end{tabular}

Data are presented as mean \pm SD or $n(\%)$ unless otherwise stated. P: participants; Non-P: nonparticipants; BL: baseline; FU: follow-up. "\#: number of valid cases may vary between variables; ${ }^{\top}$ : defined as current smokers and ex-smokers who stopped smoking <2 yrs prior to BL; ${ }^{+}$: defined as indicated in table 1 ; ${ }^{\text {s: }}$ geometric mean (geometric SD); ${ }^{f}$ : Phadiatop ${ }_{\mathbb{B}}$ (Phadia, Uppsala, Sweden) test used on a total of $1,130 \mathrm{P} .{ }^{*}: \mathrm{p}<0.05$ versus non-P (same sex and exposure); ${ }^{\# \#: ~} \mathrm{p}<0.05$ versus reference workers (same sex).

with subjects invited by letter (fig. 1). Analyses for differences between woodworkers invited outside the factories compared to woodworkers invited at the factories revealed significantly higher percentages of asthma symptoms (16.6 versus $12.3 \%$; $\mathrm{p}<0.01)$ and wheeze $(20.6$ versus $15.6 \%$; $\mathrm{p}<0.01)$ at baseline and, for female woodworkers, a higher prevalence of hay fever (16.8 versus $8.7 \%$; $\mathrm{p}<0.05$ ), but no differences in other respiratory symptoms (bronchitis and daily coughing) and smoking habits.

For reference workers who were contacted outside the factories versus reference workers still at the factories, higher percentages of asthma symptoms (19.4 versus $10.3 \%$; $<<0.01)$, wheeze (29.2 versus $14.0 \% ; \mathrm{p}<0.01)$, hay fever (19.6 versus $10.0 \%$; $\mathrm{p}<0.01)$ and smoking (61.6 versus $50.2 \% ; \mathrm{p}=0.02)$ were found.

A significant decrease in wood dust exposure was seen during the 6-yr follow-up period and has been reported earlier [12, 22]. In brief, the geometric mean (geometric SD) overall exposure at baseline was $0.9(2.1) \mathrm{mg} \cdot \mathrm{m}^{-3}$, compared to $0.6(1.6) \mathrm{mg} \cdot \mathrm{m}^{-3}$ at follow-up, with no significant differences in geometric mean at baseline and follow-up between males and females in the follow-up group. The median (range) cumulative exposure among woodworkers in the cohort was $3.6(0-7.6) \mathrm{mg} \cdot \mathrm{m}^{-3} \cdot \mathrm{yrs}$. For males and females, the cumulative exposures were $3.8(0-7.6)$ and $3.3(0-6.9) \mathrm{mg} \cdot \mathrm{m}^{-3} \cdot \mathrm{yrs}$, respectively.

Low levels of monoterpenes and formaldehyde were found. The geometric mean (geometric SD) overall monoterpene exposure was $7.0(2.8) \mathrm{mg} \cdot \mathrm{m}^{-3}$. The highest levels were observed for $\alpha$-pinene. The median (range) exposure for formaldehyde was $0.05(0.03-0.2) \mathrm{mg} \cdot \mathrm{m}^{-3}$.

In order to make the present results comparable, approximated incidence rates (IRs) were calculated based on the cumulative incidence of respiratory symptom during the follow-up period (mean 6.4 yrs) and total follow-up time of the individuals included in the analyses. Figure 2 presents the approximated IRs of respiratory symptoms for woodworkers and reference workers stratified by sex. Higher cumulative incidence proportions of current asthma and chronic bronchitis were suggested for female woodworkers compared to female reference workers.

In table 3 , the results of adjusted logistic regression of the cumulative incidence proportion of respiratory symptoms and hay fever for woodworkers versus reference workers are presented.

For females, positive but nonsignificant associations between woodwork and asthma were found. Furthermore, significant positive associations for daily coughing and bronchitis and negative associations for hay fever were revealed for female workers. In general, smoking was associated with both asthma and bronchitis. Including cumulative smoking in pack-years during follow-up alone did not change the estimates. Hay fever at baseline as a proxy of atopy was included in all models, and was positively associated with all symptoms except for bronchitis. In models in which bronchitis was the outcome, asthma symptoms at baseline were included, showing a positive association between asthma symptoms and development of bronchitis. Analyses were repeated excluding hay fever or asthma symptoms from the models (data not shown), revealing almost identical ORs of association between exposure and symptoms.

Atopic status at follow-up was included in the models for the subpopulation for which Phadiatop results were available and was, in general, found to be a predictor of respiratory 

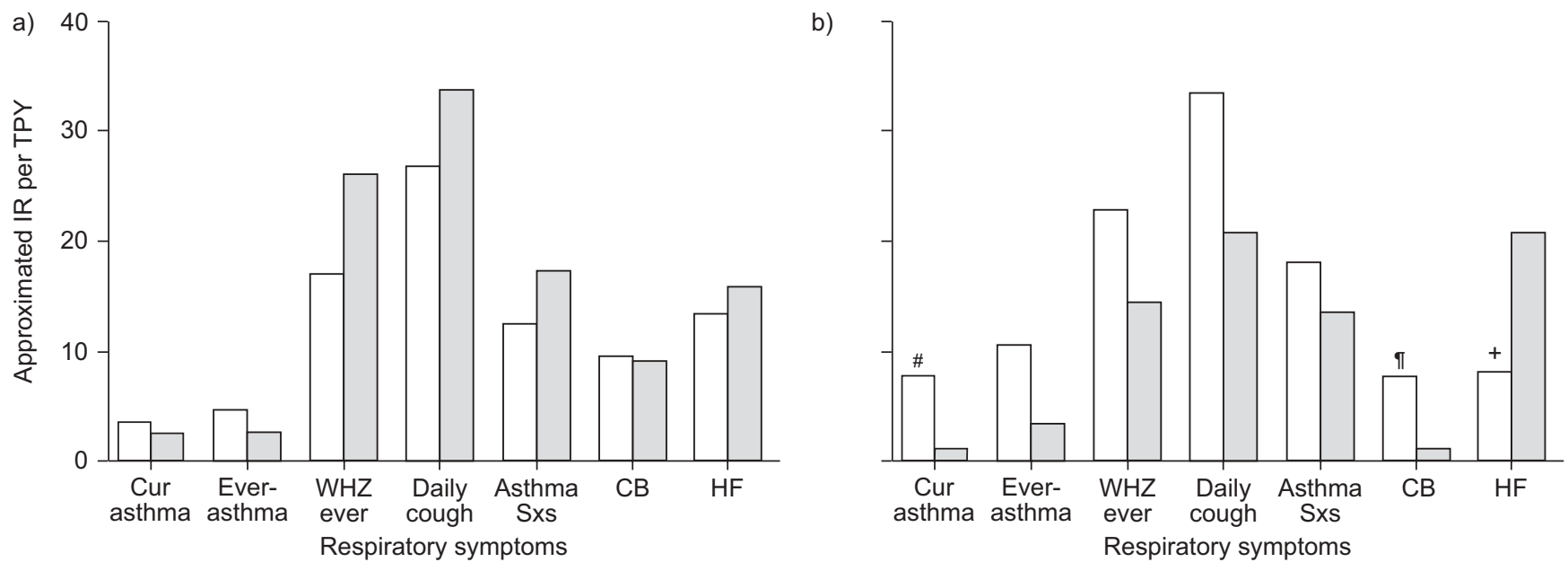

FIGURE 2. Approximated incidence rate (IR) of respiratory symptoms for woodworkers ( $\square$ ) and reference workers ( $\square$ ) in: a) males; and b) females. Approximated IRs were estimated based on the cumulative incidence of symptoms and the mean time to follow-up among workers not showing specific symptom at baseline. TPY: 1,000 person-yrs; Cur: current; WHZ: wheeze; Sxs: symptoms; CB: chronic bronchitis; HF: hay fever. ${ }^{\#}: 7.58$ (1.08-328); ": 7.37 (1.02-323); ${ }^{+}: 0.36$ (0.14-0.87) for odds ratio (95\% confidence interval) cumulative incidence proportion versus reference workers.

symptoms, but inclusion of atopy resulted only in minor changes in the OR.

The preceding analyses were performed for the cumulative incidence proportion of respiratory symptoms in cases in which the specific respiratory symptom was absent at baseline. In order to asses the influence of the general respiratory health status of the baseline cohort on the follow-up analyses, the analysis was repeated after excluding all with respiratory symptoms at baseline (fig. 3). Associations between exposure and symptoms were strengthened for female workers, with significant associations between being a woodworker and the cumulative incidence proportion of asthma symptoms, everwheeze and daily coughing, with an odds ratio (OR) (95\% confidence interval (CI)) of 11.3 (1.4-96.8), 5.9 (1.2-29.9) and 5.5 (1.9-16.3), respectively.
Adjusted logistic regression models of exposure levels (quartiles of baseline exposure among woodworkers) and cumulative incidence proportion of respiratory symptoms were performed. Reference workers were assigned to the lowest exposure category. Among female workers, positive doseresponse relations were revealed between exposure and daily coughing, as well as chronic bronchitis, with an OR (95\% CI) of 3.8 (1.5-9.7) and $6.0(1.2-28.8)$, respectively, in the most exposed group (fig. 4). A negative association between baseline exposure and cumulative incidence proportion of hay fever was suggested for female workers, ORs ranging 0.38-0.19 showing a significant negative trend $(\mathrm{p}<0.05)$.

In order to examine the association between cumulative wood dust exposure and cumulative incidence proportion of respiratory symptoms, logistic regression models of cumulative

TABLE 3 Association between cumulative incidence proportion of respiratory symptoms in the cohort and being a woodworker ${ }^{\#}$

\begin{tabular}{|c|c|c|c|c|c|c|c|}
\hline & Current asthma & Asthma ever & Wheeze ever & Asthma symptoms & Daily coughing" & Bronchitis & Hay fever \\
\hline Male workers ${ }^{+} n / N$ & $25 / 1146$ & $32 / 1099$ & $110 / 978$ & $83 / 1050$ & $139 / 804$ & $50 / 862$ & $91 / 1043$ \\
\hline Woodworkers ${ }^{\S}$ & $1.50(0.34-6.51)$ & $1.90(0.44-8.12)$ & $0.73(0.40-1.33)$ & $0.75(0.39-1.45)$ & $0.82(0.46-1.47)$ & $1.33(0.46-3.83)$ & $0.82(0.42-1.60)$ \\
\hline Smokers ${ }^{f}$ & $1.04(0.46-2.35)$ & $0.85(0.41-1.72)$ & $4.21(2.70-6.57)$ & $2.04(1.28-3.24)$ & $2.47(1.69-3.60)$ & $1.80(0.99-3.28)$ & $0.88(0.57-1.37)$ \\
\hline Hay fever at $\mathrm{BL}^{\# \#}$ & $4.41(1.89-10.3)$ & $2.60(1.13-5.95)$ & $1.47(0.79-2.71)$ & $1.82(0.98-3.39)$ & $1.47(0.87-2.49)$ & $0.76(0.31-1.89)$ & \\
\hline Asthma symptoms at $\mathrm{BL}^{\bullet \bullet}$ & & & & & & $2.98(1.48-6.00)$ & \\
\hline Smokers $f$ & $2.46(0.62-9.78)$ & $4.13(1.13-15.1)$ & $3.36(1.54-7.35)$ & $2.49(1.13-5.50)$ & $2.69(1.31-5.51)$ & $12.3(1.53-99.3)$ & $1.44(0.63-3.30)$ \\
\hline Hay fever at $\mathrm{BL}^{\# \#}$ & $2.82(0.68-11.7)$ & $2.04(0.52-7.99)$ & $1.21(0.42-3.46)$ & $1.37(0.48-3.90)$ & $1.52(0.57-4.05)$ & $1.35(0.26-7.03)$ & \\
\hline Asthma symptoms at $\mathrm{BL}^{\text {थथ }}$ & & & & & & $1.96(0.46-8.32)$ & \\
\hline
\end{tabular}

Data are presented as odds ratio (95\% confidence interval) unless otherwise indicated. BL: baseline. ${ }^{*}$ : by logistic regression analysis, with adjustment for confounders and stratified by sex; ${ }^{\prime}$ : age included in model (five age groups); ${ }^{+}$: with incident symptom/individuals included lacked symptom at BL; ${ }^{\text {s. }}$ versus reference workers; f: versus nonsmokers (newer smokers and ex-smokers who had stopped smoking $>2$ yrs prior to BL study); ${ }^{\# \#: ~ v e r s u s ~ n o ~ h a y ~ f e v e r ~ a t ~ B L ; ~ " ~}{ }^{\prime}$ versus no asthma symptoms (as defined in table 1) at BL. 

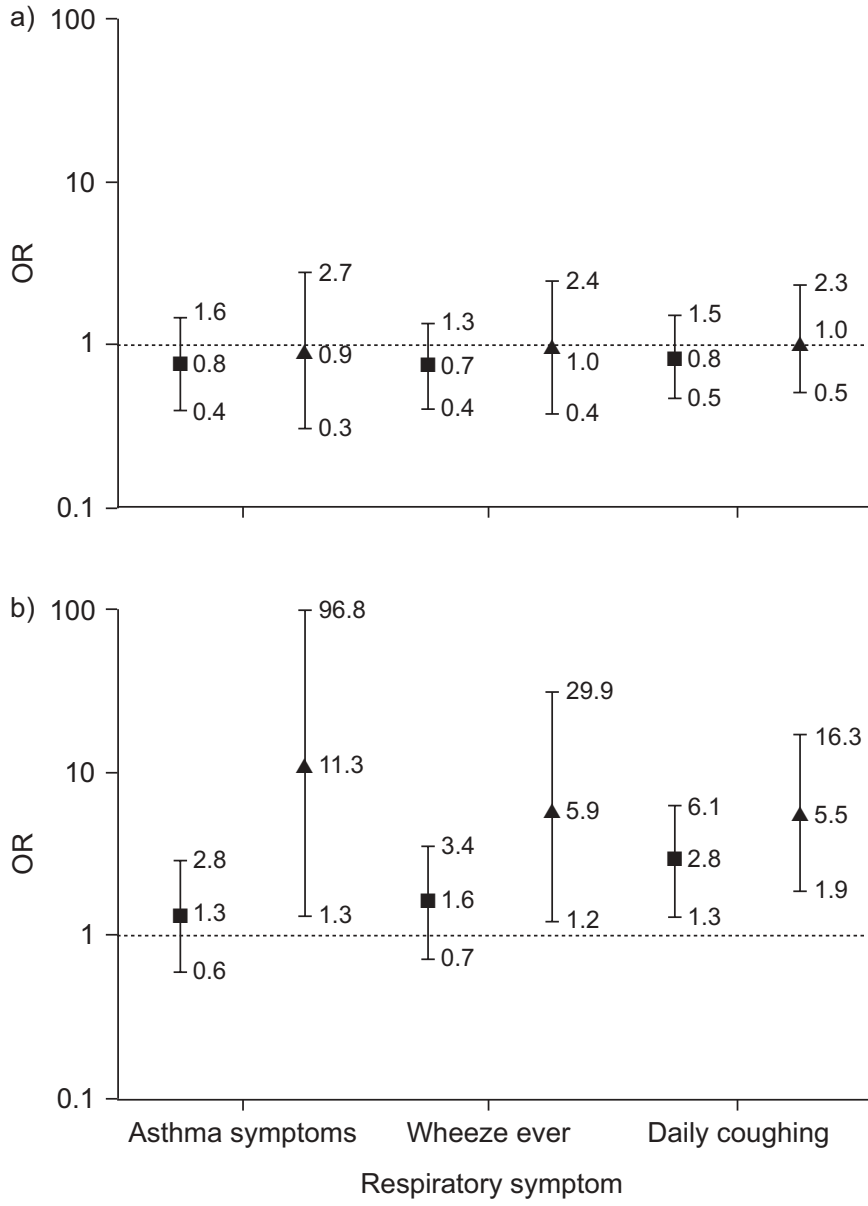

FIGURE 3. Association between cumulative incidence proportion of respiratory symptoms ( $\mathbf{\square}$ : new specific symptom, when symptom absent at baseline; $\boldsymbol{\Lambda}$ new specific symptom, when all symptoms absent at baseline) in the cohort and being a woodworker analysed by logistic regression analysis in: a) males; and b) females. The baseline respiratory symptoms included were current asthma, everasthma, ever-wheeze, daily coughing, chronic bronchitis and asthma symptoms, as defined in table 1. All analyses were adjusted for smoking, age and baseline hay fever. Vertical bars represent $95 \%$ confidence intervals (…....... odds ratio of 1 ).

exposure (four groups based on quartiles of exposure), time (in years) of exposure during follow-up (four groups) and time (in years) exposed at baseline (four groups) stratified by sex and with adjustment for smoking, age and baseline hay fever were analysed, in general showing no association between cumulative exposure indices and incidence of lower respiratory symptoms. For female workers, analyses of cumulative incidence proportion of hay fewer as an end-point, however, suggested decreasing ORs across cumulative exposure groups (three groups, with the two highest collapsed), with an OR $(95 \%$ CI) of $0.62(0.20-1.91)$ and $0.15(0.02-1.11)$ for medium and high exposure, respectively, and across groups based on exposure duration, with ORs ranging $0.43-0.17$. Tests for trend revealed significant negative trends $(\mathrm{p}<0.05)$.

In order to further examine the association between atopy and development of respiratory symptoms in relation to exposure, logistic regression was performed on atopy and development of respiratory symptoms stratified by wood dust exposure.
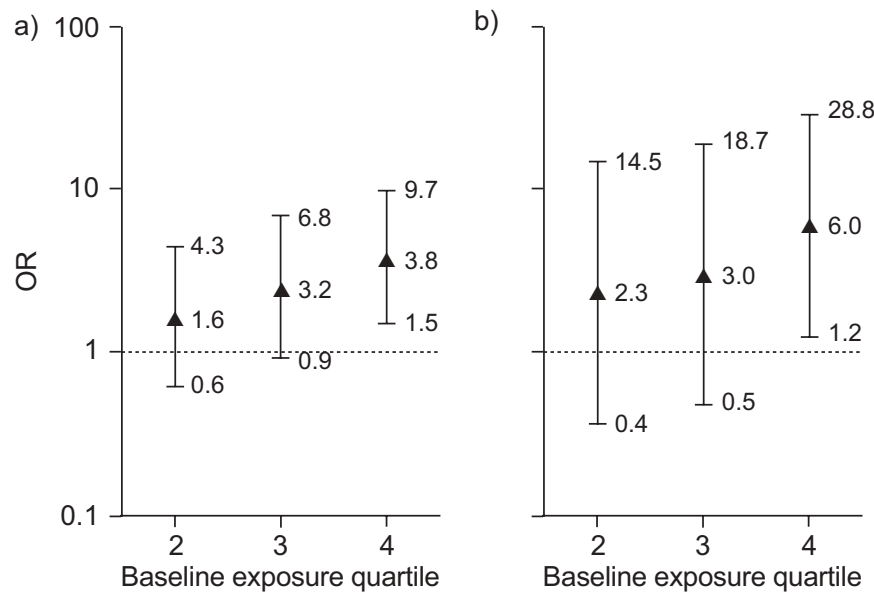

FIGURE 4. Association between cumulative incidence proportion of respiratory symptoms and baseline wood dust exposure quartile in female workers analysed by logistic regression for: a) daily coughing ( $p$-value for trend $<0.01$ ); and b) chronic bronchitis (adjusted for baseline asthma symptoms; p-value for trend $=0.02)$. The lowest exposure quartile $\left(1:<0.70 \mathrm{mg} \cdot \mathrm{m}^{-3}\right)$, including reference workers, was the reference category $\left(2: 0.70-0.94 \mathrm{mg} \cdot \mathrm{m}^{-3} ; 3: 0.95-1.32 \mathrm{mg} \cdot \mathrm{m}^{-3} ; 4\right.$ $\left.>1.32 \mathrm{mg} \cdot \mathrm{m}^{-3}\right)$. All analyses were adjusted for smoking, age and baseline hay fever. Vertical bars represent $95 \%$ confidence intervals (…...... odds ratio of 1 ).

Atopy and asthma were positively associated for woodworkers with an OR (95\% CI) ranging 3.2 (1.3-7.6) (current asthma) to 1.7 (1.0-2.8) (ever-wheeze). This association was not seen for reference workers (table 4). Analyses were repeated using hay fewer at baseline as a proxy of baseline atopy and largely revealed the same results; however, the data did not permit analysis of the reference workers.

Generally, logistic regression analyses of cumulative incidence proportion of respiratory end-points stratified by atopy (measured at follow-up) or hay fever at baseline could not be performed due to lack of power. However, analyses of nonatopic workers and workers without hay fever at baseline showed the same results as analyses without restriction.

For female woodworkers, leaving the furniture industry was associated with increased cumulative incidence proportion of asthma, with ORs ranging 13.1 (2.5-65.9) (ever-asthma) to 1.8 (0.6-5.9) (ever-wheeze). No associations were seen for male woodworkers (table 4).

Linear regression analyses at baseline and follow-up, stratified by sex and adjusted for age and smoking, revealed significant negative associations between percentage predicted FEV1 and asthma symptoms, ever-asthma, daily coughing and bronchitis, with regression coefficients at follow-up ranging $-8.14 \%$ pred FEV1 $(\mathrm{p}<0.00)$ (asthma symptoms) to $-4.15 \%$ pred FEV1 $(p<0.00)$ (daily coughing), with no clear difference between males and females. The analyses were repeated using logistic regression in order to explore the association between asthma symptoms and decreased FEV1 $(<80 \%$ pred). A higher proportion of subjects with asthma symptoms showed a decreased FEV1 compared to nonasthmatics, with an OR $(95 \% \mathrm{CI})$ of $3.4(2.2-5.4)$ and $2.8(1.04-7.4)$ for males and females, respectively. 


\begin{tabular}{|c|c|c|c|c|c|c|c|}
\hline \multirow[t]{2}{*}{ TABLE 4} & \multicolumn{7}{|c|}{$\begin{array}{l}\text { Adjusted logistic regression models of association between cumulative incidence proportion of respiratory symptoms } \\
\text { and atopy (stratified by exposure) and current occupation in the wood industry among woodworkers stratified by sex }\end{array}$} \\
\hline & & Current asthma & Asthma ever & Wheeze ever & Asthma symptoms & Daily coughing & Bronchitis \\
\hline \multicolumn{2}{|c|}{ Wood workers $^{\#} n / N$} & $23 / 863$ & $32 / 829$ & $98 / 740$ & $89 / 791$ & $115 / 609$ & $23 / 666$ \\
\hline \multicolumn{2}{|c|}{ Atopy at $\mathrm{FU}^{+}$} & $3.15(1.30-7.59)$ & $3.10(1.47-6.54)$ & $1.65(0.98-2.80)$ & $1.87(1.11-3.16)$ & $1.35(0.81-2.26)$ & $0.67(0.27-1.64)$ \\
\hline \multicolumn{2}{|c|}{ Reference workers ${ }^{\#} n / N$} & $3 / 179$ & $5 / 171$ & 23/156 & $24 / 172$ & $21 / 125$ & $4 / 123$ \\
\hline \multicolumn{2}{|c|}{ Male wood worker ${ }^{\#, 9} n / N$} & 20/902 & $27 / 868$ & $79 / 775$ & $60 / 830$ & $106 / 638$ & $40 / 691$ \\
\hline \multicolumn{2}{|c|}{ Employed outside wood industry at $\mathrm{FU}^{\S}$} & $1.69(0.63-4.53)$ & $1.11(0.44-2.80)$ & $1.04(0.57-1.88)$ & $0.90(0.46-1.75)$ & $0.96(0.57-1.63)$ & $0.71(0.29-1.75)$ \\
\hline \multicolumn{2}{|c|}{ Female wood worker ${ }^{\#, 9} \mathrm{n} / \mathrm{N}$} & $6 / 168$ & 9/157 & $17 / 141$ & $13 / 147$ & $21 / 115$ & $5 / 132$ \\
\hline \multicolumn{2}{|c|}{ Employed outside wood industry at FU $U^{\S}$} & $3.02(0.56-12.9)$ & $13.10(2.51-68.87)$ & $1.83(0.56-5.97)$ & $4.65(1.37-15.8)$ & $0.96(0.29-2.86)$ & NP \\
\hline
\end{tabular}

Data are presented as odds ratio (95\% confidence interval) unless otherwise indicated. Age (two groups) and smoking (smokers comprise current smokers and exsmokers who had stopped smoking <2 yrs prior to baseline) were included in all models. FU: follow-up; NP: not possible. ${ }^{\#}$ : with incident symptom/individuals included lacked symptom at baseline; ${ }^{\natural}$ : hay fever at baseline included in model; ${ }^{+}$: versus nonatopic individuals; ${ }^{\S}$ : versus employment in wood industry at FU.

Additionally, significant negative associations between percentage predicted FEV1 at baseline and incidence of respiratory symptoms were revealed. For example, the regression coefficient was $-5.52 \%$ pred FEV1 $(p<0.00)$ for incidence of asthma symptoms, with no significant differences between males and females.

Linear regression analyses of association between relative annual change in FEV1 and baseline asthma symptoms, adjusted for age, height, smoking and change in weight, revealed an accelerated decline in FEV1 among workers reporting asthma symptoms at baseline, with coefficients of $-0.27 \%$ annual change in FEV1 $(p<0.01)$, with no difference between males and females.

Additionally, a negative association between incident cases of self-reported ever-asthma and decline in FEV1 with a regression coefficient of $-0.46 \%$ annual change in FEV1 $(p<0.05)$, with no difference between males and females, was found.

\section{DISCUSSION}

To the present authors' knowledge, the current study is the first industry-specific follow-up study in the wood industry investigating the association between wood dust exposure and the occurrence of new respiratory symptoms. The present results suggest that females, but not males, develop respiratory symptoms (coughing and bronchitis), and asthma, despite the relatively low wood dust level.

The present study reports approximated IRs based on cumulative incidence proportions of self-reported ever-asthma ranging 2.6-10.6 per 1,000 person-yrs (TPY) (fig. 2). Approximated IRs decreased to $0-5.8$ per TPY when analyses were restricted to persons without any respiratory symptom at baseline. In comparison to most population studies, these approximated IRs among adults are relatively high. In a recent follow-up study involving the Scandinavian Countries, TOREN et al. [23] reported IRs of self-reported ever-asthma of 2.2 per TPY, ranging 1.5-2.9 per TPY and decreasing to 1.5 per TPY after exclusion of subjects with respiratory symptoms at baseline.

Two Finnish follow-up studies performed from populationbased registers on reimbursement for asthma medication and job status have reported elevated relative risks (RRs) for incident asthma cases for woodworking in both males and females: KARJALAINEN et al. [8] found a RR (95\% CI) of 1.6 (1.5$1.8)$ in males and $1.6(1.4-1.8)$ in females, and HeIKKILÄ et al. [9] of $1.5(1.2-1.8)$ in males and $1.5(1.2-1.7)$ in females, with no clear dose-response. These studies are hardly comparable with the present study due to large differences in study design and accuracy of exposure estimates. Job status is a crude proxy for wood dust exposure with no direct information on exposure level. However, for furniture workers, who had been shown previously to be relatively highly exposed to wood dust [10], HeIKKILÄ et al. [9] did not report elevated RRs for incident asthma cases.

In an epidemiological setting, the annual incidence of red cedar asthma was estimated from cross-sectional data to be 4$5 \%$ based on information on four patients leaving the plant [7]. For other wood types, no incidence data are available.

For females, positive associations were found between cumulative incidence proportion of symptoms and baseline dust exposure, whereas no clear associations were revealed for cumulative dust exposure. This suggests that mean baseline exposure better reflects the exposure of interest. One reason could be that it reflects peak exposures more efficiently. Earlier studies in the dry wood industry using cumulative exposure indices have reported conflicting results. SHAMSSAIN [24] reported an increased prevalence of respiratory symptoms with increasing duration of employment, whereas BOHADANA et al. [4] found no associations between cumulative wood dust exposure and prevalence of respiratory symptoms among woodworkers with a median cumulative exposure of $110 \mathrm{mg} \cdot \mathrm{m}^{-3} \cdot \mathrm{yrs}$.

The overall participation rate in the follow-up study was $67 \%$, which may potentially cause skewing of risk estimates due to selection bias. A higher percentage of workers contacted at the factories participated compared with those invited by letter (fig. 1). This indicates that people who had left the industry did not have the same incitement to participate. The fact that a substantial proportion of the woodworkers had left the industry might contribute to healthy-worker selection in the 
cohort, since persons experiencing respiratory symptoms might be more prone to leaving the industry. No difference was found in baseline respiratory symptoms and mean dust level between participants and drop-outs, and also no significant difference in participation rate between woodworkers and controls. However, woodworkers and reference workers invited outside the factories showed a higher prevalence of asthma symptoms and hay fever (female woodworkers only) at baseline compared to workers invited at the factories. A higher incidence of self-reported everasthma and asthma symptoms was also found among female workers who had left the industry for employment elsewhere. This difference could not be explained by a difference in wood dust levels, smoking or symptoms at baseline, and indicates that female woodworkers who experience symptoms are more inclined to leave the industry, creating healthy-worker selection.

Alternatively, it could be suggested that workers with symptoms who had left the industry were more inclined to participate than subjects without symptoms. Since female participants outside the furniture industry, in general, reported the same prevalence of respiratory symptoms at baseline as drop-outs, it seems unlikely that selection of symptomatic workers into the study took place. Indeed, a negative association had previously been found between coughing and seniority in the wood industry, indicating selection away from the industry [3]. In the present study, a negative association between hay fever and exposure was suggested for female workers, which could also support selection of symptomatic workers out of the industry. Therefore, if selection bias exist in the present study, it is likely to have influenced the result towards a weaker association between wood dust exposure and health outcome.

At baseline, the present definition of asthma symptoms was validated against objective measures of airway obstruction [16]. In addition, analyses revealed associations between baseline FEV1 and incidence of respiratory symptoms, as well as associations between symptoms and FEV1 at baseline and follow-up, and between decline in FEV1 and development of self-reported asthma. This supported decreased FEV1 as a risk factor for the development of respiratory symptoms and underlined an objective basis for the respiratory symptoms recorded in the present questionnaire.

The measured inhalable wood dust concentrations in the present study were low, with a geometric mean of $0.94 \mathrm{mg} \cdot \mathrm{m}^{-3}$ at baseline and $0.60 \mathrm{mg} \cdot \mathrm{m}^{-3}$ at follow-up. It has recently been estimated that 560,000 workers in the EU may be exposed to levels of $>5 \mathrm{mg} \cdot \mathrm{m}^{-3}$ and that a concentration of $2 \mathrm{mg} \cdot \mathrm{m}^{-3}$ may be exceeded by 2 million workers [10]. This suggests that the Danish furniture industry is at the very low end of the wood dust exposure distribution in Europe.

Atopy was found to be associated with asthma among woodworkers, but not among controls, which supports the earlier results from the baseline study [25] suggesting atopy to be an important modifier of the association between asthma and wood dust exposure. However, including hay fewer as a proxy for atopy did not modify the results. A follow-up study among subjects with western red cedar asthma concluded that atopy was not a modifying factor [26]. Earlier results do not suggest immunoglobulin-E-mediated allergy to be a major course of wood-dust-induced asthma, at least not for frequently used wood types, such as pine and beech [27], or red cedar asthma [26].

Animal studies have shown that abietic acid, the major constituent of pine resin, can produce lytic damage to alveolar, tracheal and bronchial epithelial cells [28], and it has been associated with occupational asthma in other industries. In the present study, abietic acid content was not analysed.

Terpenes, naturally occurring substances in pine and other coniferous trees, have been documented to cause irritation of mucous membranes and increase bronchial responsiveness [29]. Levels of total terpenes found in the present study were low, with a geometric mean of $7.0 \mathrm{mg} \cdot \mathrm{m}^{-3}$, well below the Danish occupational exposure limit of $140 \mathrm{mg} \cdot \mathrm{m}^{-3}$.

Processing of plywood and fibreboard may cause exposure to formaldehyde, and asthma symptoms have been documented among woodworkers exposed to formaldehyde alone or in combination with wood dust. A health-based occupational exposure limit of $0.15 \mathrm{mg} \cdot \mathrm{m}^{-3}(0.12 \mathrm{ppm})$ has been recommended in the Netherlands [30]. In the present study, low levels of formaldehyde were found, with a geometric mean of $0.05 \mathrm{mg} \cdot \mathrm{m}^{-3}$. This level is also low compared to a recent Finnish study reporting an overall GM (geometric SD) of 0.17 $(1.83) \mathrm{mg} \cdot \mathrm{m}^{-3}$ in furniture factories processing mainly medium-density fibreboard and $0.10(1.75) \mathrm{mg} \cdot \mathrm{m}^{-3}$ in factories processing mainly coniferous wood [31].

The present study indicates females to be more susceptible to wood dust exposure than males with regard to the development of bronchitis, coughing and possibly asthma. This study has previously revealed dose-response relations between wood dust exposure and an accelerated annual decline in lung function among female woodworkers [12]. Likewise, MATHESON et al. [32] found significant associations between exposure to biological dust and chronic obstructive bronchitis, and COPD in females but not in males. Several population studies have reported a higher incidence of asthma among females $[23,33]$ and a greater susceptibility to the development of lung function impairment in relation to tobacco exposure [33]. The causes of females being more prone to developing asthma, respiratory symptoms and lung function impairment are not fully understood. Various explanations have been presented, e.g. airway size, hormonal factors and social factors; in addition, a greater tendency for physicians to diagnose asthma in females and COPD in males has been mentioned $[23,33]$.

A sex-related difference of the cough reflex has also been suggested, with females having a more sensitive cough reflex than males when tested with capsaicin. The proposed mechanism was greater sensitivity of the sensory receptors of the respiratory tract [34].

In conclusion, the present results suggest that females, but not males, have an increased incidence of respiratory symptoms despite the low exposure level in the Danish furniture industry. The present study supports that wood dust levels well below current occupational exposure limits for wood dust, e.g. $5 \mathrm{mg} \cdot \mathrm{m}^{-3}$ in the EU, cause respiratory symptoms. 


\section{REFERENCES}

1 International Agency for Research on Cancer. IARC Monographs on the Evaluation of Carcinogenic Risks to Humans. Vol. 62. Wood Dust and Formaldehyde. Lyon, International Agency for Research on Cancer, 1995.

2 Demers PA, Teschke K, Kennedy SM. What to do about softwood? A review of respiratory effects and recommendations regarding exposure limits. Am J Ind Med 1997; 31: 385-398.

3 Schlünssen V, Schaumburg I, Taudorf E, et al. Respiratory symptoms and lung function among Danish woodworkers. J Occup Environ Med 2002; 44: 82-98.

4 Bohadana AB, Massin N, Wild P, et al. Symptoms, airway responsiveness, and exposure to dust in beech and oak wood workers. Occup Environ Med 2000; 57: 268-273.

5 Schlünssen V, Sigsgaard T, Schaumburg I, et al. Cross-shift changes in FEV1 in relation to wood dust exposure: the implications of different exposure assessment methods. Occup Environ Med 2004; 61: 824-830.

6 Chan-Yeung M, Barton GM, Maclean L, et al. Occupational asthma and rhinitis due to western red cedar (Thuja plicata). Am Rev Respir Dis 1973; 108: 1094-1102.

7 Chan-Yeung M, Ashley MJ, Corey P, et al. A respiratory survey of cedar mill workers. I. Prevalence of symptoms and pulmonary function abnormalities. J Occup Med 1978; 20: 323-327.

8 Karjalainen A, Kurppa K, Martikainen R, et al. Work is related to a substantial portion of adult-onset asthma incidence in the Finnish population. Am J Respir Crit Care Med 2001; 164: 565-568.

9 Heikkilä P, Martikainen R, Kurppa K, et al. Asthma incidence in wood-processing industries in Finland in a register-based population study. Scand J Work Environ Health 2008; 34: 66-72.

10 Kauppinen T, Vincent R, Liukkonen T, et al. Occupational exposure to inhalable wood dust in the member states of the European Union. Ann Occup Hyg 2006; 50: 549-561.

11 Jacobsen G. Respiratory diseases and exposure in the Danish furniture industry: a 6 year follow-up. Phd thesis. University of Aarhus, Aarhus, Denmark, 2007.

12 Jacobsen G, Schlünssen V, Schaumburg I, et al. Longitudinal lung function decline and wood dust exposure in the furniture industry. Eur Respir J 2008; 31: 334-342.

13 Definition and classification of chronic bronchitis for clinical and epidemiological purposes. A report to the Medical Research Council by their Committee on the Aetiology of Chronic Bronchitis. Lancet 1965; 1: 775-779.

14 Burney PG, Luczynska C, Chinn S, et al. The European Community Respiratory Health Survey. Eur Respir J 1994; 7: 954-960.

15 Sigsgaard T, Hjort C, Omland $\varnothing$, et al. Respiratory health and allergy among young farmers and non-farming rural males in Denmark. The SUS study. J Agromedicine 1997; 4: 63-78.

16 Schlünssen V. Asthma and other respiratory diseases among workers in the furniture industry occupationally exposed to wood dust. Phd Thesis. University of Aarhus, Aarhus, Denmark, 2001.

17 Cantani A, Ferrara M, Barbieri C, et al. Evaluation of new test (Phadiatop) for the screening of respiratory allergic disorders in children. Ann Allergy 1990; 64: 158-161.

18 Vinzents PS. A passive personal dust monitor. Ann Occup Hyg 1996; 40: 261-280.

19 Schlünssen V, Vinzents PS, Mikkelsen AB, et al. Wood dust exposure in the Danish furniture industry using conventional and passive monitors. Ann Occup Hyg 2001; 45: 157-164.

20 Eriksson K. Evaluation of a diffusive sampler for air sampling of monoterpenes. Analyst 1994; 119: 85-89.

21 Levin J-O, Lindahl R, Andersson K. Research Report. Determination of Formaldehyde Using the SKC UMEx 100 (Cat. No. 500-100) Diffusive Sampler. Eighty Four, SKC, Inc., 2006.

22 Schlünssen V, Jacobsen G, Erlandsen M, et al. Determinants of wood dust exposure in the Danish furniture industry results from two cross-sectional studies 6 years apart. Ann Occup Hyg 2008; 52: 227-238.

23 Toren K, Gislason T, Omenaas E, et al. A prospective study of asthma incidence and its predictors: the RHINE study. Eur Respir J 2004; 24: 942-946.

24 Shamssain MH. Pulmonary function and symptoms in workers exposed to wood dust. Thorax 1992; 47: 84-87.

25 Schlünssen V, Schaumburg I, Heederik D, et al. Indices of asthma among atopic and non-atopic woodworkers. Occup Environ Med 2004; 61: 504-511.

26 Chan-Yeung M. Immunologic and nonimmunologic mechanisms in asthma due to western red cedar (Thuja plicata). J Allergy Clin Immunol 1982; 70: 32-37.

27 Skovsted TA, Schlünssen V, Schaumburg I, et al. Only few workers exposed to wood dust are detected with specific IgE against pine wood. Allergy 2003; 58: 772-779.

28 Ayars GH, Altman LC, Frazier CA, et al. The toxicity of constituents of cedar and pine woods to pulmonary epithelium. J Allergy Clin Immunol 1989; 83: 610-618.

29 Malmberg PO, Rask-Andersen A, Larsson KA, et al. Increased bronchial responsiveness in workers sawing Scots pine. Am J Respir Crit Care Med 1996; 153: 948-952.

30 Dutch Expert Committee on Occupational Standards. Formaldehyde. Health-Based Recommended Occupational Exposure Limit. 2003/02OSH, 1-127. The Hague, Health Council of the Netherlands, 2003.

31 Priha E, Pennanen S, Rantio T, et al. Exposure to and acute effects of medium-density fiber board dust. J Occup Environ Hyg 2004; 1: 738-744.

32 Matheson MC, Benke G, Raven J, et al. Biological dust exposure in the workplace is a risk factor for chronic obstructive pulmonary disease. Thorax 2005; 60: 645-651.

33 de Marco R, Locatelli F, Sunyer J, et al. Differences in incidence of reported asthma related to age in men and women. A retrospective analysis of the data of the European Respiratory Health Survey. Am J Respir Crit Care Med 2000; 162: 68-74.

34 Dicpinigaitis PV, Rauf K. The influence of gender on cough reflex sensitivity. Chest 1998; 113: 1319-1321. 